\title{
Cryptic pockets repository through pocket dynamics tracking and metadynamics on essential dynamics space: application to $\mathrm{Mcl}-1$
}

Mohammed Benabderrahmane, ${ }^{\dagger}$ Ronan Bureau, ${ }^{\dagger}$ Anne Sophie Voisin-Chiret ${ }^{\dagger}$ and Jana Sopkova-de Oliveira Santos $\dagger^{\dagger *}$

$\dagger^{\prime}$ Normandy University, UNICAEN, Centre d'Etude et Recherche sur le Médicament de Normandie (CERMN), Caen, France 


\section{Supporting Figures}
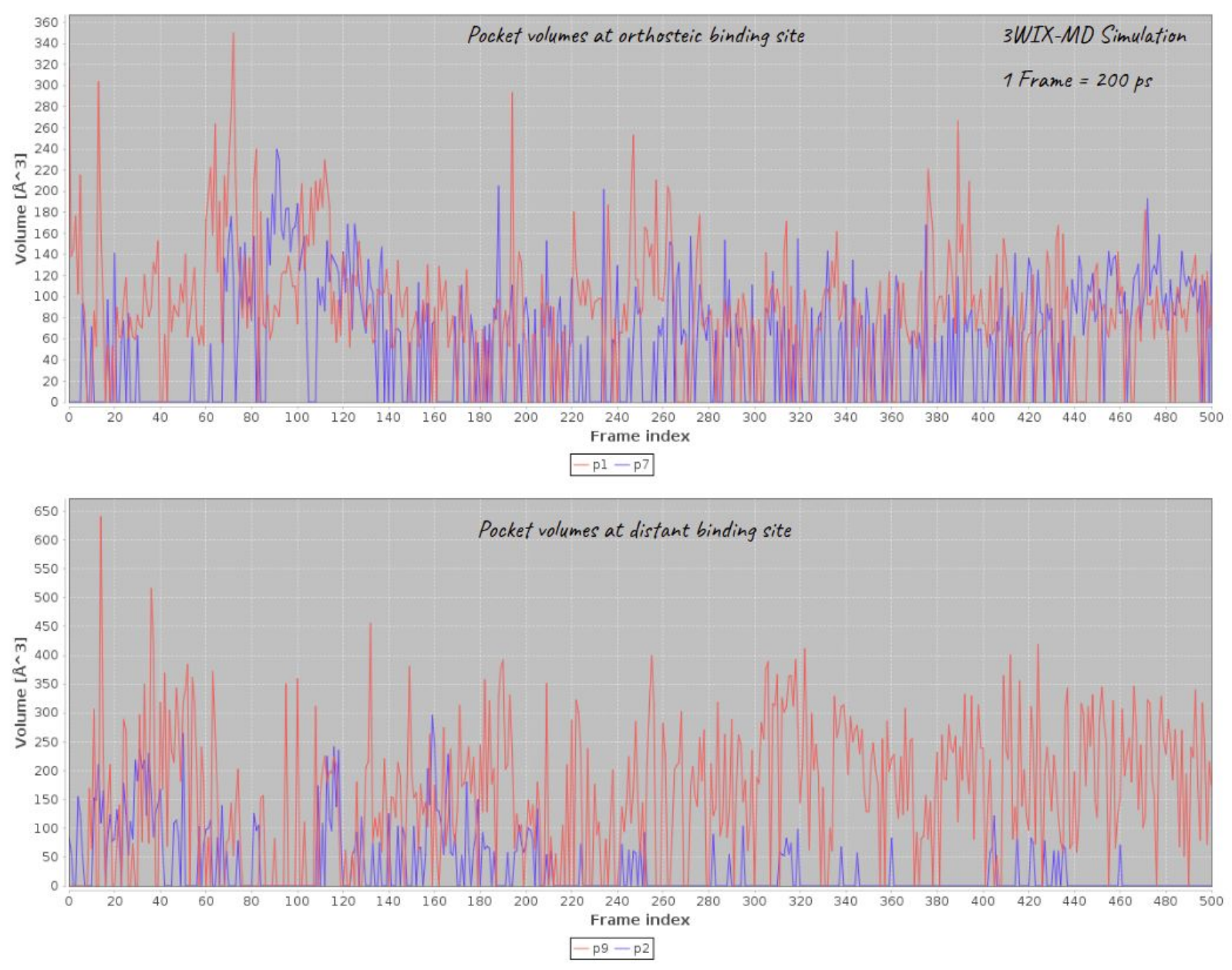

Figure S1. Pocket volume time series for the 3WIX-MD simulation 

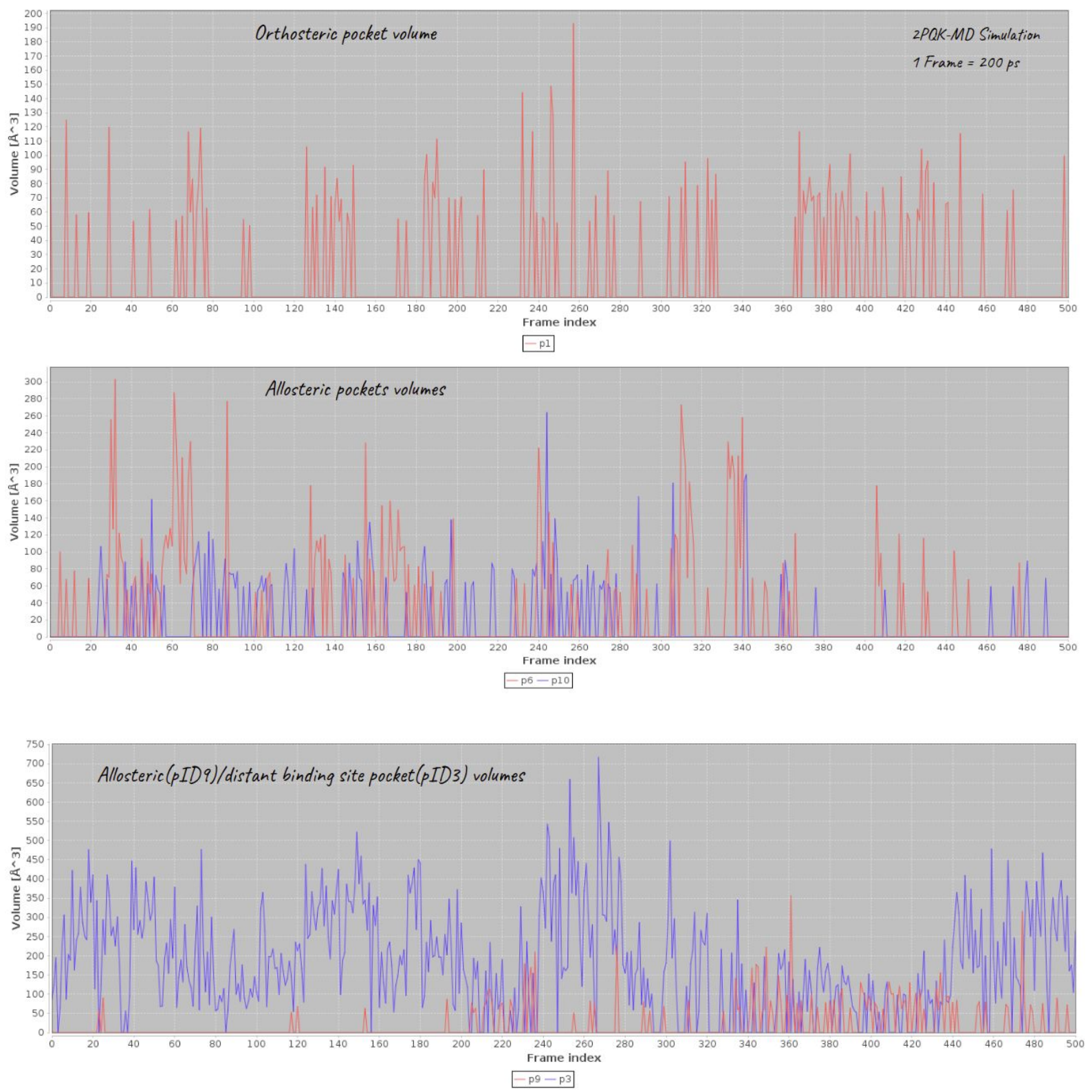

Figure S2. Pocket volumes time series for the $2 \mathrm{PQK}-\mathrm{MD}$ simulation 

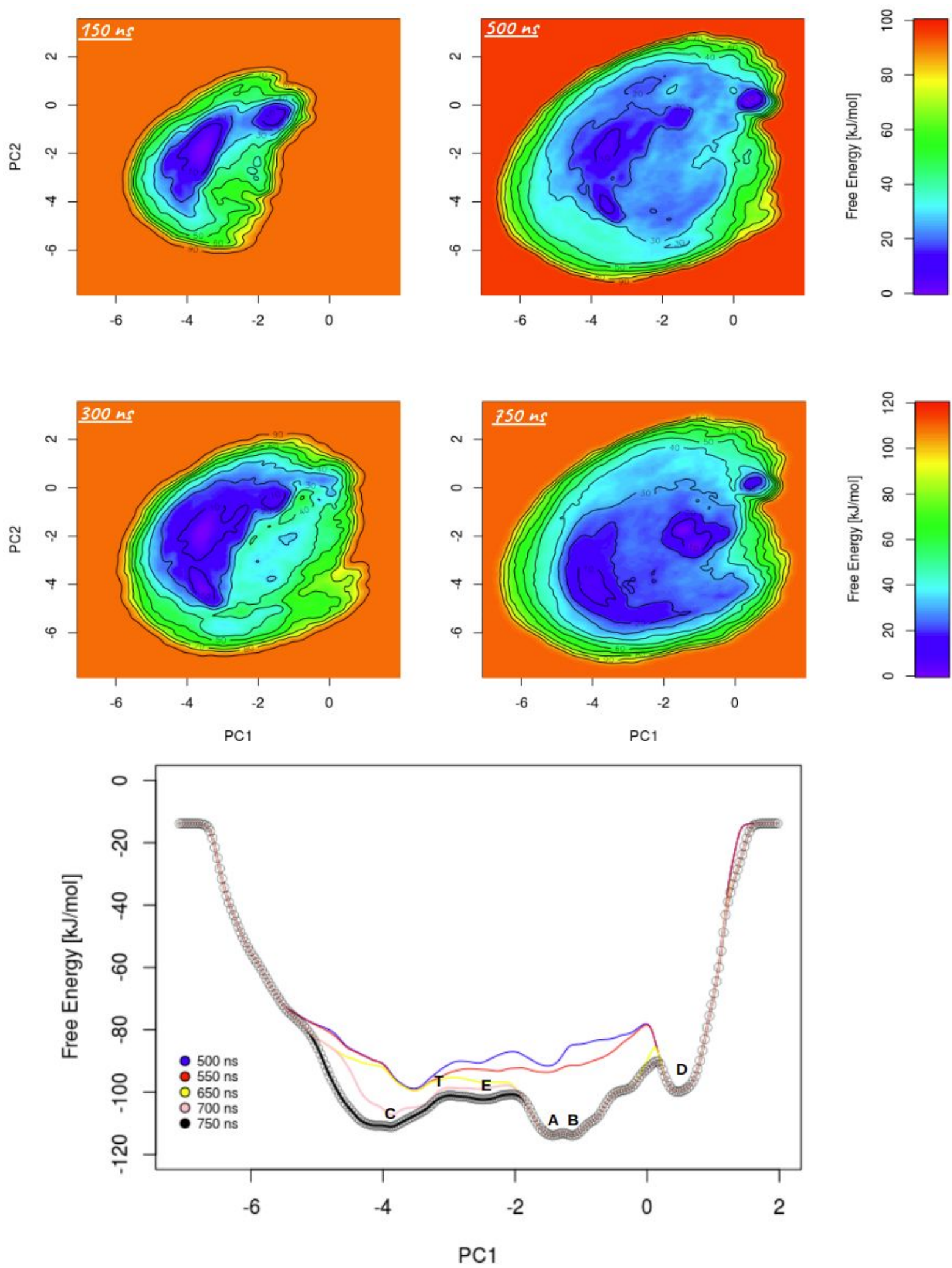

Figure S3. Qualitative assessment of the FES convergence. A. FES evolution during time. B. Time evolution of the free energy profile along the first collective variable (PC1) 


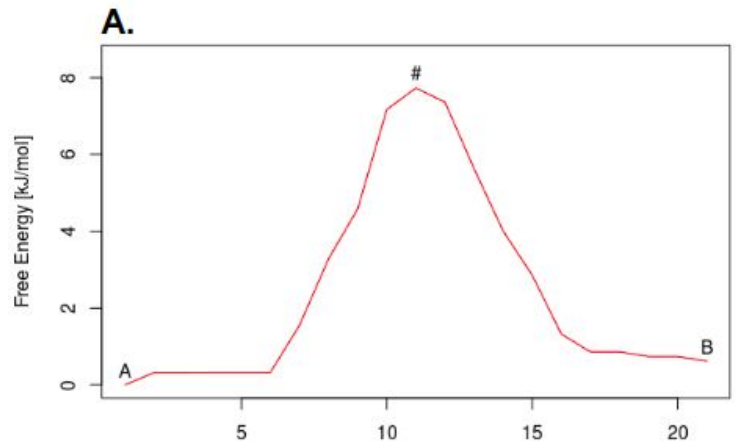

B.
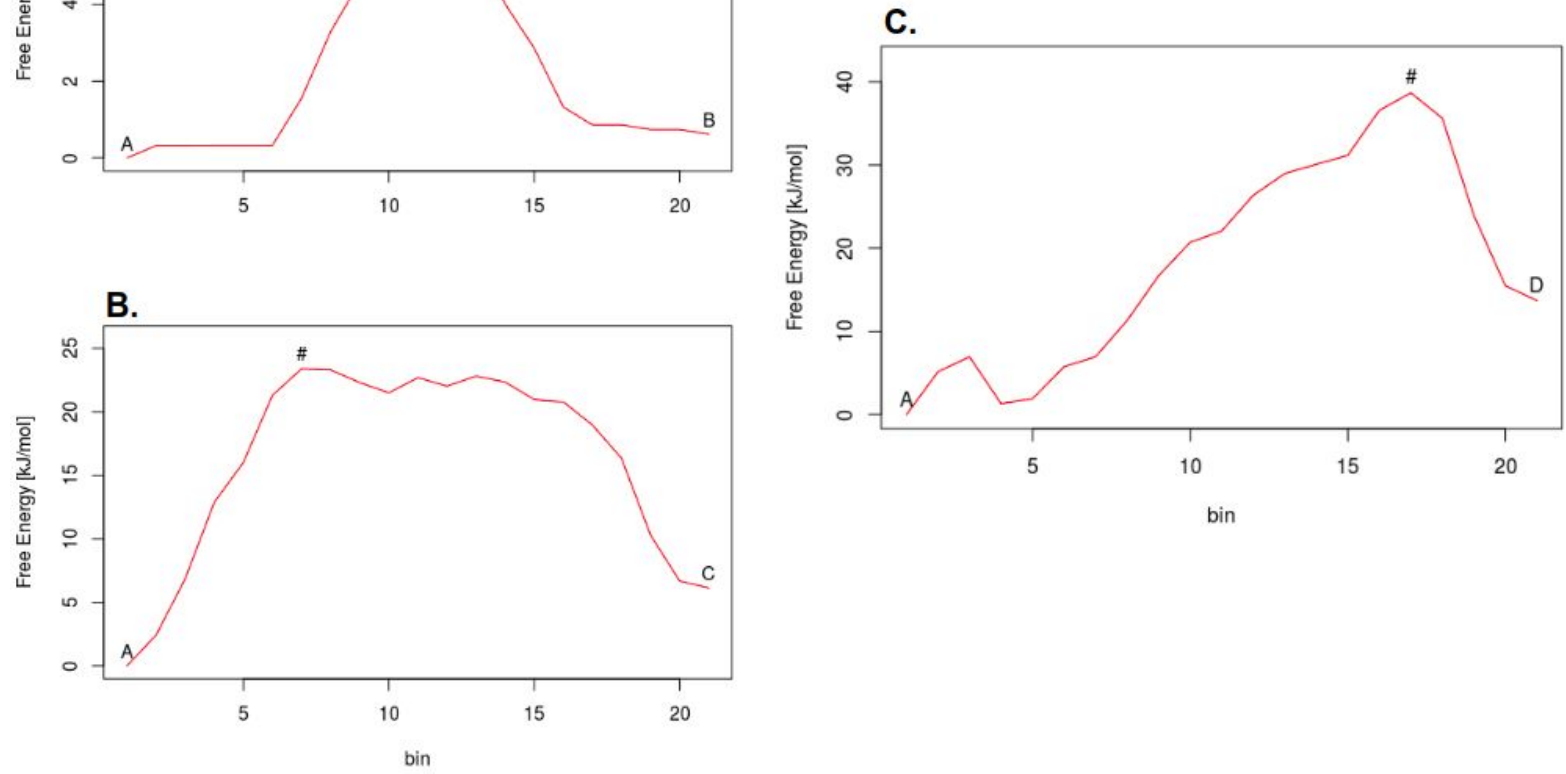

Figure S4. Free energy barriers computed via the Nudged Elastic Band (NEB) Analysis. A. Transition A -> B : 8 kJ/mol ; B. Transition A -> C : $23 \mathrm{~kJ} / \mathrm{mol}$; C. Transition A -> D : 33 $\mathrm{kJ} / \mathrm{mol}$ 


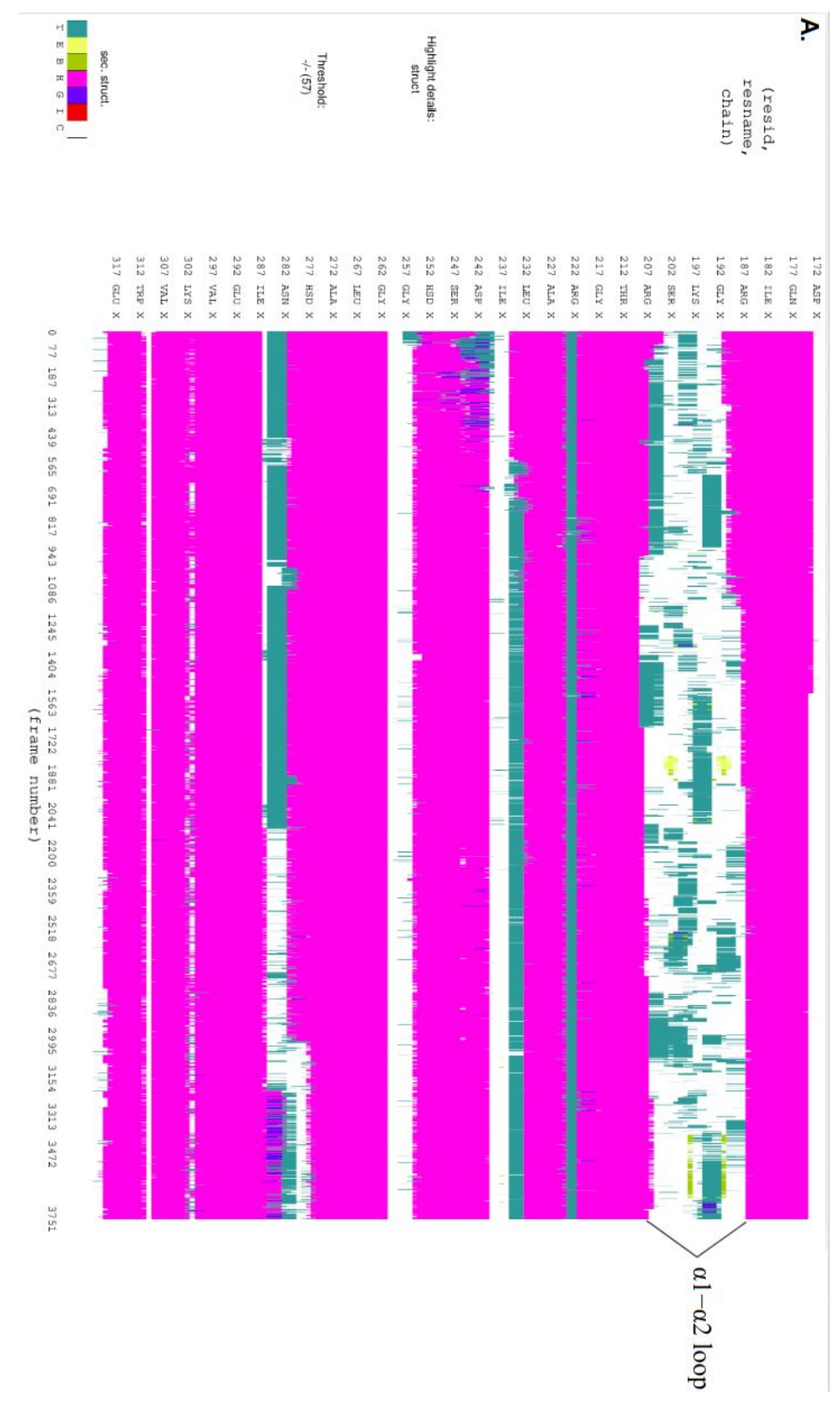




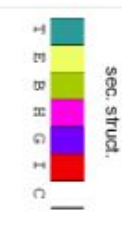

$$
\text { n!l| }
$$

\section{篦意}

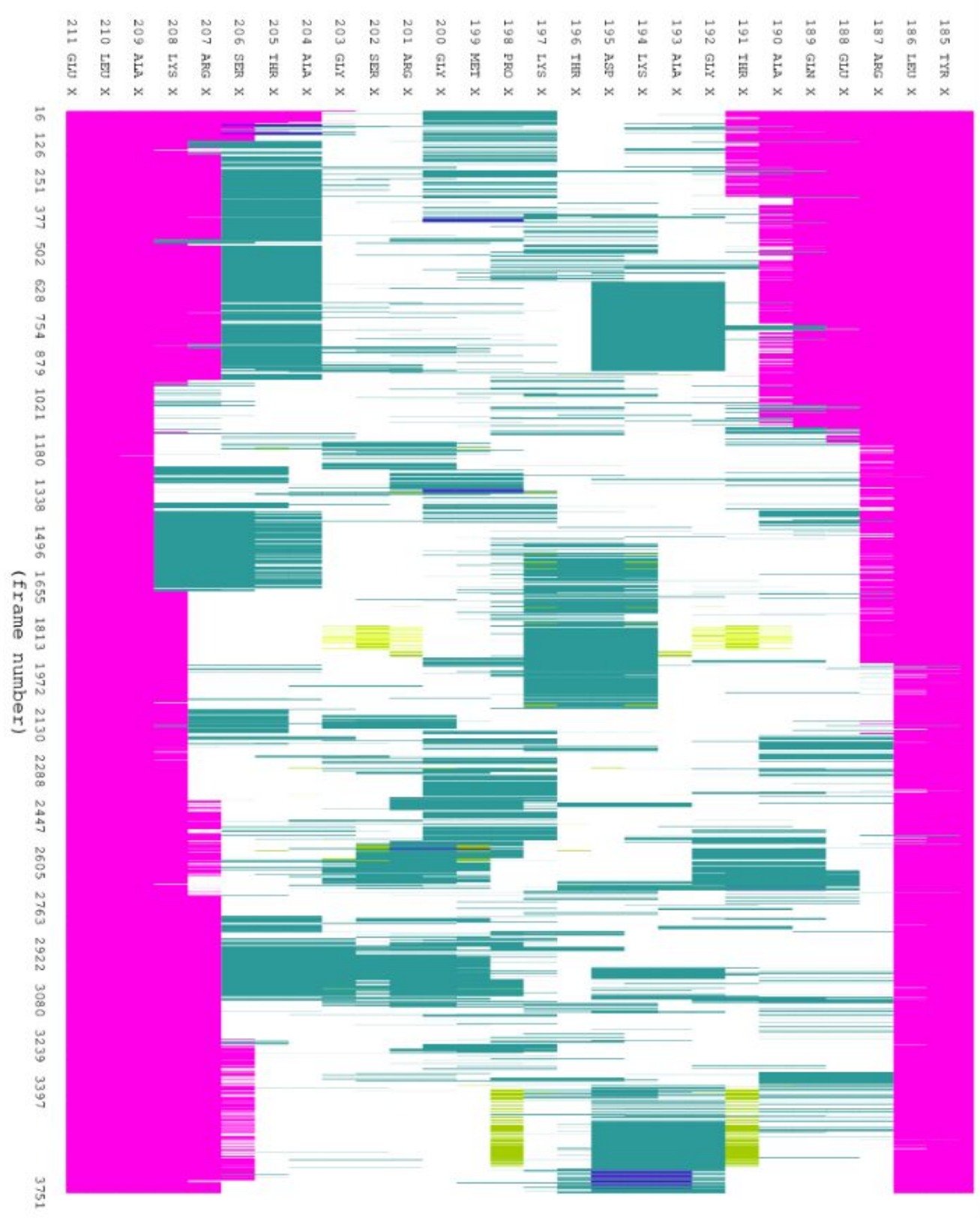

Figure S5. The dynamics of the $\alpha 1-\alpha 2$ loop are highly influenced by the conformational transitions at the C-terminal end of the $\alpha 1$ helix (residues Arg187 Glu188 Gln189 Ala190 Thr191) and the N-terminal end of $\alpha 2$ helix (Gly203 Ala204 Thr205 Ser206 Arg207 Lys208). Secondary structures (computed using STRIDE) evolution during the Metadynamics simulation. A. For all the protein; B. For the $\alpha 1-\alpha 2$ loop. 


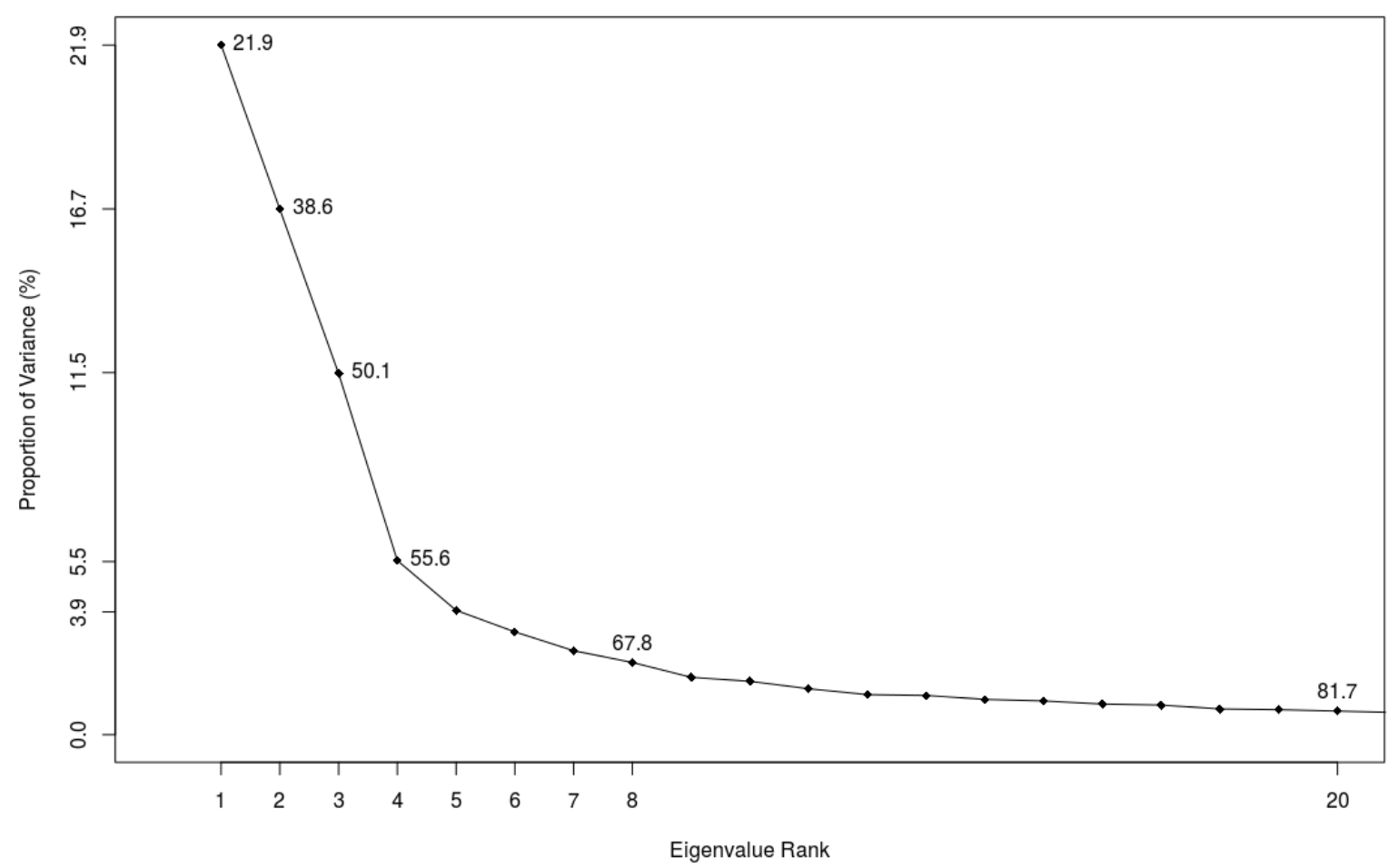

Figure S6. Eigen spectrum plot (scree plot) for the 2PQK-MD simulation. The first and the second eigenvector account for $\sim 39 \%$ of the variability (accumulated variance) in the trajectory.

The Eigen spectrum plot shows that the two eigenvectors (extracted from the 2PQK-MD simulation) used as collective variables space account for $\sim 39 \%$ of the accumulated variance in the trajectory. Using the "Elbow" criterion, it would be more convenient to choose a large number of eigenvectors (up to 4 here) but this will require a much more sampling effort to converge the free-energy surface. In the classical form of the well-tempered Metadynamics, a rule of thumb is to choose two separate collective variables. However, other flavors of the Metadynamics (ex. Bias-exchange Metadynamics) allow the use of more than two collective variables, but still, the interpretation of the free-energy surface resulting from such schemes is far from being intuitive.

Two natural ways to enhance the amount of variance explained by the eigenvectors are to compute the eigenspace from a "merged" trajectory starting from different conformations or to use a much longer simulation. Other dimensionality reduction methods that do not assume the linearity of the protein dynamics (unlike the PCA), such as ISOMAPs might be used as well. 


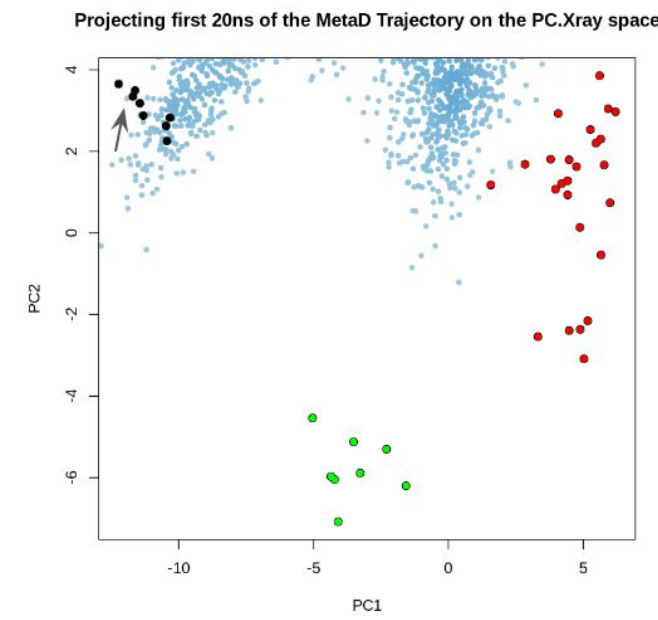

Projecting 100-120 ns of the MetaD Trajectory on the PC.Xray space

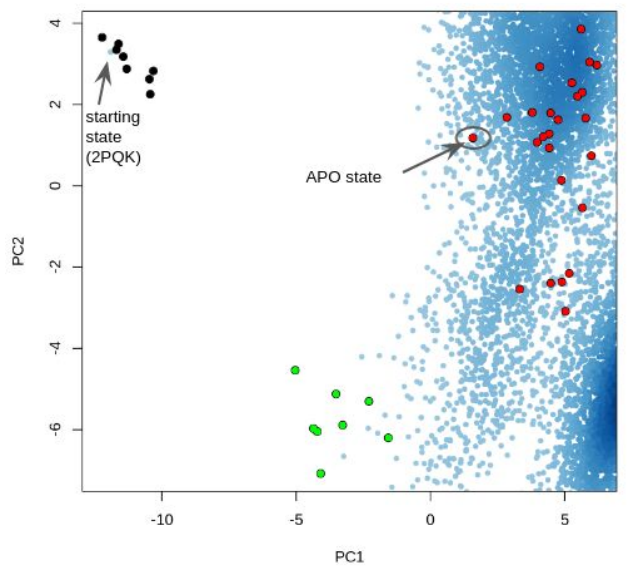

Projecting 280-300 ns of the MetaD Trajectory on the PC.Xray space

Projecting 600-620 ns of the MetaD Trajectory on the PC.Xray space
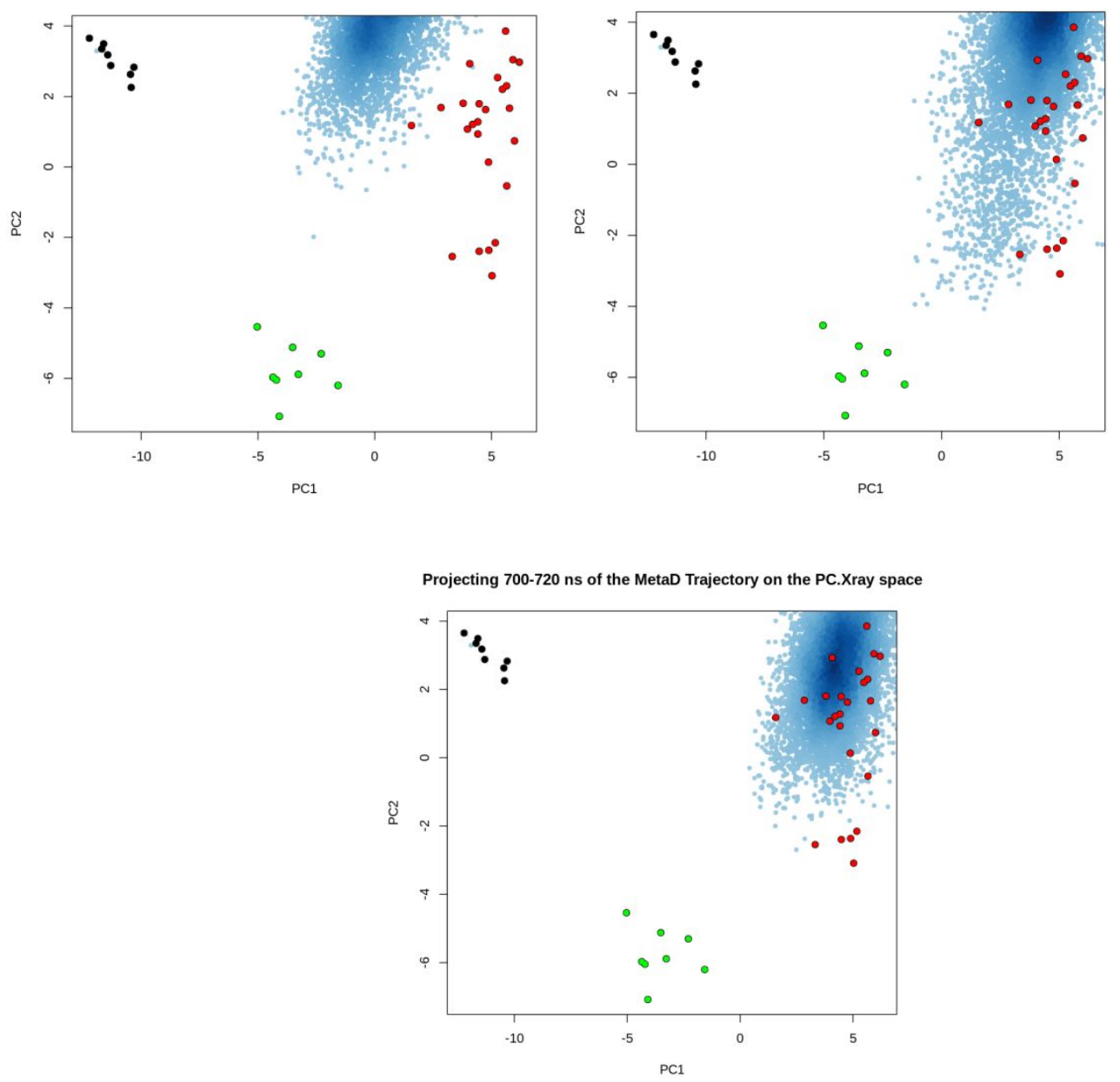

Figure S7. Projection of MetaD conformational space on Mcl-1 X-ray (PCs) space. Black and green dots represent peptide-bound conformations. Red dots represent synthetic-ligand bound conformations, except the rounded APO state. 
A. $\min A$
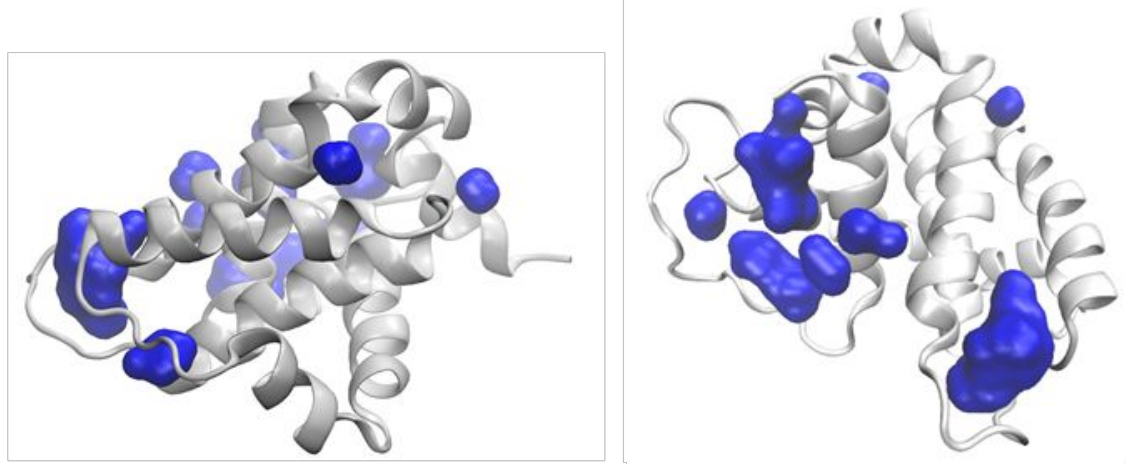

B. $\min B$
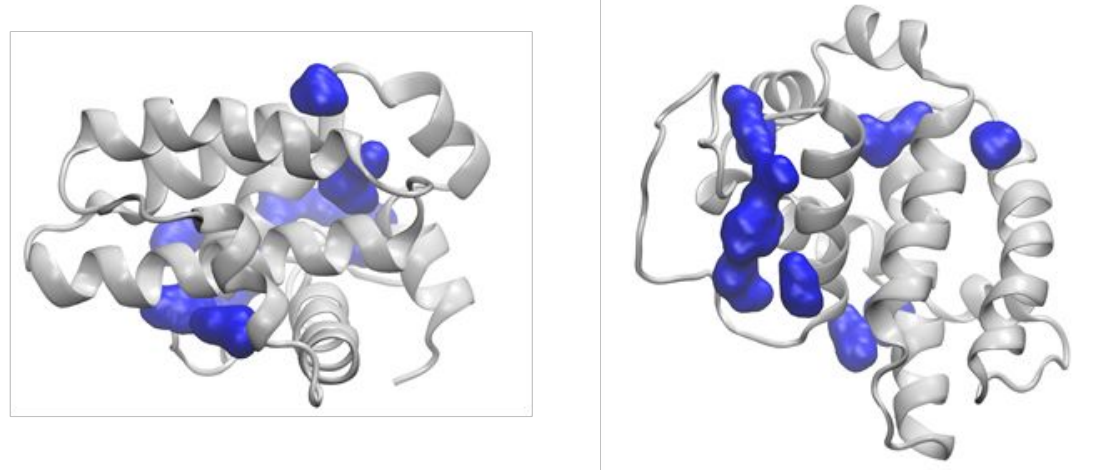

C. $\min C$
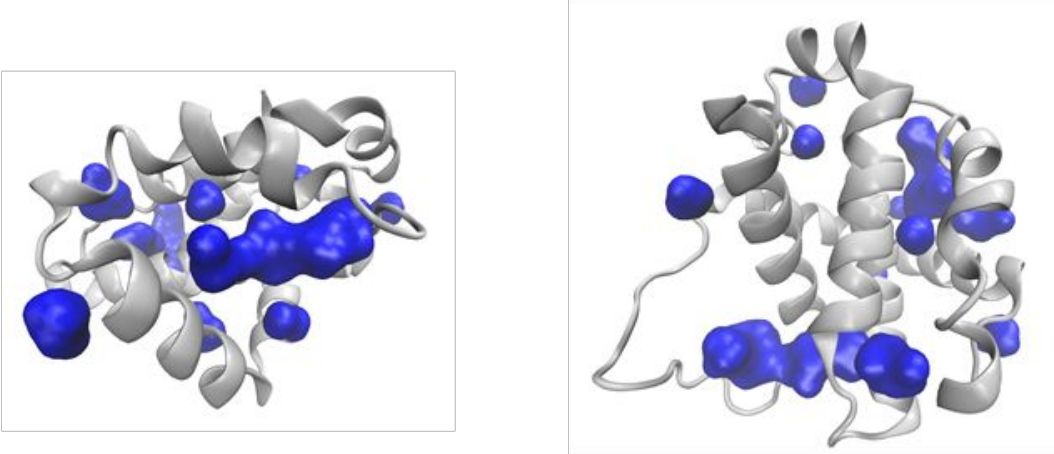

D. $\min D$
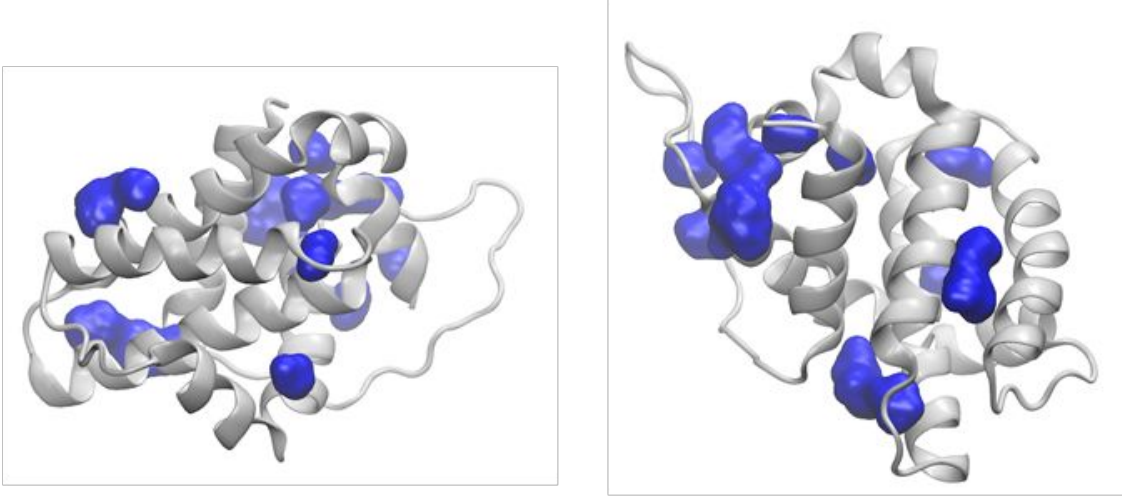

E. $\min E$
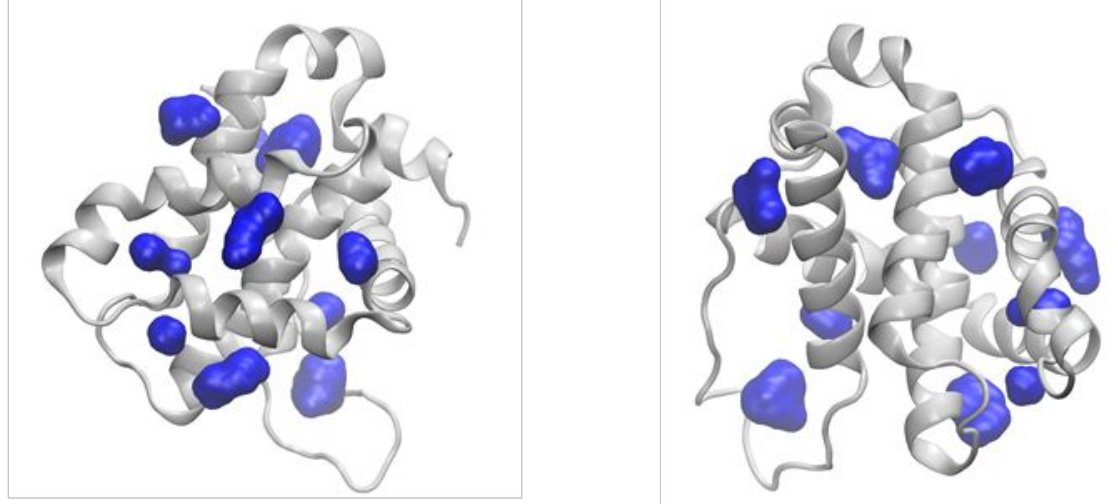
Figure S8. The cryptic pockets detected by NanoShaper (Biki Technologies) from different minima observed along the Metadynamics simulations. 


\section{Supporting tables}

A. PDB ID 2MHS

\begin{tabular}{|c|c|c|}
\hline pID & Volume $\left(\AA^{3}\right)$ & Residues \\
\hline 0 & 50 & Arg184 Asp195 Thr196 Lys197 Met199 Leu210 \\
\hline 1 & 92 & Met170 Glu171 Tyr175 Asp296 Val 299 Arg300 Arg303 \\
\hline 2 & 210 & $\begin{array}{c}\text { Phe254 Asp256 Gly257 Val258 Thr259 Asn260 Trp261 Arg263 Ile264 } \\
\text { Lys302 Trp305 Gln309 }\end{array}$ \\
\hline 3 & 37 & Asp218 Gln221 Arg222 Glu225 Phe273 Lys276 \\
\hline
\end{tabular}

\section{B. PDB ID 3WIX}

\begin{tabular}{|c|c|c|}
\hline pID & Volume $\left(\AA^{3}\right)$ & Residues \\
\hline 0 & 99 & Ile183 Arg184 Arg187 Asp195 Lys197 Glu288 \\
\hline 1 & 319 & $\begin{array}{r}\text { Ala227 Phe228 Met231 Leu235 Leu246 Val249 Met250 Val253 } \\
\text { Phe254 Arg263 Thr266 Leu267 Phe270 Gly271 Val274 Leu290 Ile294 }\end{array}$ \\
\hline 2 & 81 & Arg176 Met199 Gly200 Arg201 Ser202 Gly203 Ser206 \\
\hline 3 & 42 & Lys208 Glu211 Thr212 Arg215 Val316 His320 \\
\hline 4 & 50 & Lys194 Asp195 Thr207 Leu210 Glu211 Arg214 \\
\hline
\end{tabular}

\section{PDB ID 2PQK}

\begin{tabular}{|c|c|c|}
\hline pID & Volume $\left(\AA^{3}\right)$ & Residues \\
\hline 0 & 113 & Met231 Leu235 Val249 Val253 Thr266 Leu267 Phe270 \\
\hline 1 & 85 & Arg176 Leu179 Glu180 Ser183 Arg184 Arg187 Asp195 Arg201 \\
\hline 2 & 86 & Arg184 Asp195 Thr196 Lys197 Pro198 Ser202 Arg207 Leu210 \\
\hline 3 & 35 & Met250 Phe254 Ile264 Leu267 Ile268 Ile294 Val297 Leu298 \\
\hline
\end{tabular}

Tables S1. Pocket residues and volumes from experimental structures computed via Nanoshaper. A. PDB ID 2MHS; B. PDB ID 3WIX; C. PDB ID 2PQK 
A. 3WIX-MD

\begin{tabular}{|c|c|c|c|}
\hline pID & $\begin{array}{c}\text { Avg.Vol } \\
\left(\AA^{3}\right)\end{array}$ & Residues & Pocket position \\
\hline 9 & 156 & Glu188 Leu210 Arg207 Lys194 Asp195 Arg184 Arg187 Ala193 & Distant binding site \\
\hline 1 & 87 & Phe254 Arg 263 Lys302 Leu267 Thr259 Ile264 Asp256 Val258 & $\begin{array}{c}\text { Upper part of orthosteric } \\
\text { binding site }\end{array}$ \\
\hline 7 & 56 & Ile237 His277 Leu232 Ile281 & $\begin{array}{c}\text { Near the allosteric binding } \\
\text { site }\end{array}$ \\
\hline 3 & 53 & Arg187 Arg184 Glu288 Ser183 Asp195 Glu188 Ala193 Lys197 & Distant binding site \\
\hline 13 & 35 & Trp312 Gln177 Asp313 Arg201 Leu174 Gly311 & Distant binding site \\
\hline 2 & 30 & Arg201 Gln177 Gln180 Gly200 Arg176 & Distant binding site \\
\hline 11 & 20 & GIn221 Lys276 Arg222 Phe273 Glu225 Asp218 & $\begin{array}{l}\text { Pocket under the } \\
\text { orthosteric binding site }\end{array}$ \\
\hline
\end{tabular}

B. 2PQK-MD

\begin{tabular}{|c|c|c|c|}
\hline pID & $\begin{array}{l}\text { Avg.Vol } \\
\qquad\left(\AA^{3}\right)\end{array}$ & Residues & Pocket position \\
\hline 3 & 177 & Leu210 Arg184 Lys 197 Arg214 Met199 Arg207 Pro198 & Distant binding site \\
\hline 4 & 78 & $\begin{array}{l}\text { Gln221 Gln189 Ala272 Ser269 Phe273 Tyr185 Lys276 Asp218 } \\
\text { Gly217 }\end{array}$ & $\begin{array}{l}\text { Pocket behind the } \\
\text { orthosteric binding site }\end{array}$ \\
\hline 6 & 27 & Leu278 Ile281 Lys238 Glu240 Cys286 & Allosteric binding site \\
\hline 9 & 19 & Lys279 Ile287 Ser285 Arg187 Thr191 Cys286 & Allosteric binding site \\
\hline 10 & 18 & Pro289 Lys244 Glu240 Val243 Cys286 Leu278 Leu290 Ser285 & Allosteric binding site \\
\hline 1 & 16 & Phe270 Val249 Met231 Hsd252 Val253 Phe228 Thr266 & Orthosteric binding site \\
\hline
\end{tabular}

Table S2. Pocket residues and average volumes for the unbiased MD simulations: A. 3WIXMD; B. 2PQK-MD. 
A. orthosteric site

\begin{tabular}{|c|c|c|}
\hline pID & $\begin{array}{c}\text { Avg.Vol } \\
\left(\AA^{3}\right)\end{array}$ & Main residues \\
\hline 9 & 31 & Phe254 Arg263 Val253 Asp256 Thr266 \\
\hline 2 & 14 & Asn282 Ser285 Leu278 Lys238 Ile281 Ile237 \\
\hline 8 & 30 & Hsd277 Leu232 Ile237 Val274 Asp236 Leu278 Lys279 Leu235 \\
\hline 26 & 15 & $\begin{array}{r}\text { Leu232 Hsd277 Ile237 } \\
\hline 11\end{array}$ \\
\hline 1 & 53 & $\begin{array}{r}\text { Lys276 Gln221 Ala272 Phe273 Tyr185 Ser269 Gln189 Asp218 } \\
\text { Glu225 Gly217 Phe228 }\end{array}$ \\
\hline
\end{tabular}

\section{B. Allosteric site}

\begin{tabular}{|c|c|c|}
\hline pID & $\begin{array}{c}\text { Avg.Vol } \\
\left(\AA^{3}\right)\end{array}$ & Main residues \\
\hline 6 & 44 & Glu188 Gln189 Arg214 Tyr185 \\
\hline 7 & 67 & $\begin{array}{r}\text { Ile287 Arg187 Lys279 Ser285 Ala275 Leu186 Glu188 Glu284 } \\
\text { Cys286 Gln283 Leu278 }\end{array}$ \\
\hline 12 & 30 & Pro289 Val243 Leu278 Cys286 Leu290 Glu240 Ile237 Ser285 \\
\hline
\end{tabular}

\section{Distant binding site}

\begin{tabular}{|c|c|c|}
\hline 17 & 68 & $\begin{array}{r}\text { Asp313 Trp312 Gln177 Ala209 Ile181 Ser206 Val316 Lys208 } \\
\text { Thr205 Arg207 Glu180 }\end{array}$ \\
\hline 3 & 40 & Glu180 Gln177 Ile181 Ser206 Thr205 Arg184 Ala204 \\
\hline 4 & 77 & $\begin{array}{r}\text { Arg184 Leu210 Arg207 Glu180 Ser206 Arg201 Ser202 Thr205 } \\
\text { Ala204 Gly203 }\end{array}$ \\
\hline 5 & 19 & Thr212 Arg215 Lys208 Glu211 Val316 Hsd320 Val321 Phe319 \\
\hline
\end{tabular}

Table S3. Pocket residues and average volumes for the Metadynamics simulation: A. Orthosteric pockets; B. Allosteric pockets; C. Distant binding site pockets. 